\title{
Conditions de vie des ménages et recours aux soins parmi les personnes âgées au Cameroun
}

Par

MVEING Séraphin

Ministère de l'Economie, de la Planification, de l'Aménagement du Territoire (MINEPAT, Cameroun) et FOMEKONG Félicien
Institut National de la Statistique, Cameroun

Résumé

Les transitions démographiques en cours en Afrique sub-Saharienne affectent progressivement la structure démographique de la population, et posent ainsi de nouvelles questions liées au vieillissement des populations. Cette étude examine le recours aux soins de santé parmi les personnes âgées, notamment la diversité des stratégies dans ce domaine et l'influence des conditions de vie des ménages. Même si les analyses, basées sur des données d'enquête (ECAM II), confirment l'importance des caractéristiques démographiques et économiques des ménages, elles mettent aussi en exergue l'importance des croyances étiologiques et la vulnérabilité généralisée des personnes âgées face à la maladie. L'étude suggère ainsi une prise de conscience accrue de la vulnérabilité de ce groupe émergent.

\section{Abstract}

Demographic transitions in sub-Saharan Africa are gradually changing the region's age structure and raising new questions related to ageing. This study investigates the diversity and factors associated with the use of health services among the elderly, with special emphasis on the influence of household characteristics and resources. Even as our analyses of ECAM II data confirm the importance of household characteristics, they also underline the relevance of local norms and belief systems, as well as the widespread vulnerability of the elderly when faced with health issues. The study thus recommends increased recognition of the vulnerability of this growing segment of the population. 


\section{Introduction}

Le vieillissement des populations est une tendance universelle qui n'épargne plus aucun continent (EGGERICKS et TABUTIN, 2001: 23). Il entraîne d'importantes mutations dans la pyramide des âges, avec une augmentation de la proportion et du nombre des personnes âgées. Selon les estimations des NationsUnies, près d'un million de personnes traversent chaque mois le seuil de 60 ans, âge considéré comme référence pour l'entrée dans la vieillesse. La même source indique qu'au Cameroun, les personnes de 65 ans et plus représentaient environ 3,6\% de la population totale du pays entre 1990 et 2000. Lorsque cette borne est réduite à 55 ans, âge moyen de départ en retraite dans différentes administrations du pays, cette proportion atteint 5,5\% (EVINA AKAM, 2003:9). Des projections des Nations Unies, il ressort que cette population augmentera dans les prochaines années avec la transition démographique en cours et du fait de l'allongement de l'espérance de vie à la naissance. Jusqu'à présent, le Cameroun comme bien d'autres pays africains, s'est préoccupé des questions relatives à la jeunesse de sa population, aux conséquences sociales, économiques et politiques de cette jeunesse et à la maîtrise de la procréation à travers la politique de parenté responsable, les questions de santé maternelle et infantile, la crise économique et la lutte contre la pauvreté et le fléau sida. Or, les changements dans les structures familiales auxquels se greffent des mutations démographiques l'allongement de la durée de vie, la baisse amorcée de la mortalité et de la fécondité, la surmortalité des adultes atteints de sida, l'émigration juvénile et l'exode ruralaffectent progressivement la structure démographique de sa population, en posant des problèmes liés au vieillissement. Les données censitaires et celles issues de nombreuses enquêtes ou projections démographiques attestent l'émergence de ce processus de vieillissement. Elles montrent aussi une augmentation de la proportion et du nombre de personnes âgées, surtout en milieu rural (BARBIER, COURADE et GUBRY, 1981-82 :107-147; J. C. BARBIER, G. COURADE et P. GUBRY, 1978). Par ailleurs, le vieillissement de la population constitue désormais un facteur de l'évolution des sociétés ou de changement social, avec des répercussions sur la crise des solidarités traditionnelles. Cette croissance de la population âgée engendre plusieurs problèmes dérivés $\mathrm{d} u$ vieillissement, parmi lesquels la demande et le recours aux soins. Comme le relève HENRARD (1992:21)

"Le vieillissement de la population s'accompagne d'une augmentation du nombre des personnes atteintes de maladies chroniques, non mortelles, plus ou moins invalidantes. Ces maladies ajoutent leur retentissement fonctionnel aux effets du vieillissement; ceci accentue le risque et la difficulté ou même l'incapacité à effectuer un certain nombre de gestes et d'activités de la vie courante. Ces incapacités, lorsqu'elles se conjuguent à un environnement défaillant, sont à l'origine de handicaps ou désavantages sociaux compromettant les six rôles de survie définis par l'Organisation Mondiale de la Santé (OMS) : indépendance physique, orientation dans le 
MVEING Séraphin, FOMEKONG Félicien: Conditions de vie des ménages et recours aux soins parmi les personnes âgées au Cameroun

temps et dans l'espace, capacité à se déplacer dans son environnement immédiat (mobilité), occupations habituelles pour l'âge et le sexe, suffisanceéconomique».

Prise dans ce sens, la vieillesse apparaît comme un handicap potentiel, d'où l'intérêt de s'interroger sur le recours des personnes âgées aux soins de santé. Les familles africaines sont aujourd'hui touchées par l'âgisme, et dans ce contexte, " on ne peut pas toujours s'attendre que les anciens soient respectés et vénérés comme il était de tradition» Andrews (1992:22). Par ailleurs, la crise économique des années 1990 et les mesures d'ajustement structurel prises par la suite par les bailleurs de fonds ont davantage précarisé les conditions de vie des populations, rendant plus difficile l'utilisation des services sociaux payants notamment ceux de santé (COURADE, 1994). Au sein des familles, les inégalités face à la santé se sont accrues en affectant spécialement les populations dépendantes, inactives et les cadets sociaux déjà vulnérables: enfants, femmes, personnes âgées (AKOTO ELIWO, M. et al, 2002). Dans ce contexte, les populations déploient de multiples stratégies pour se soigner. Celles-ci peuvent être discriminatoires selon le sexe, l'âge, le statut familial du patient et bien d'autres variables individuelles et collectives. Le manque d'intérêt accordé aux personnes âgées mérite que l'on s'y attarde pour étudier l'importance et les déterminants de leur recours aux soins de santé. En particulier, vers quels secteurs de soins recourent les personnes âgées lorsqu'elles sont malades? Quels sont les facteurs qui les y prédisposent? Quelles sont les raisons qui les motivent ? Ces questionnements sont la toile de fond de notre analyse.

\section{Contexte et considérations méthodologiques}

Comme la plupart des pays en développement, les pays africains sont engagés dans la transition démographique depuis la seconde moitié du 20ème siècle. Cette transition est caractérisée par une baisse relative de la fécondité et de la mortalité, qui a engendré une réduction des classes d'âge jeune et une augmentation des classes adultes et vieilles. Elle a été favorisée dans la plupart des cas par des politiques gouvernementales volontaristes et se déroule sur une courte période. Elle pourrait se traduire à court terme par un vieillissement accéléré de la population de ces pays contrairement aux pays développés ou le vieillissement démographique a été progressif du fait de l'étalement de la transition démographique sur une longue période. Dans ces pays, les questions de vieillissement de la population revêtent aujourd'hui un grand intérêt. Si dans la plupart des déclarations de politique de population en Afrique, les personnes âgées représentent désormais un groupe vulnérable, les études et les programmes et politiques en leur faveur sont encore rares.

Jusqu'à un passé récent, le vieillissement de la population était considéré comme un phénomène propre aux sociétés occidentales, lesquelles sont entrées plus tôt dans le processus de transition démographique. Aujourd'hui, il 
se généralise dans le monde entier sous l'effet de plusieurs facteurs sociaux, économiques, politiques, culturels, démographiques. Cette pluralité de facteurs explicatifs rend difficile la saisie du phénomène. Plus fondamentalement, la définition même des personnes du troisième âge varie d'un contexte à l'autre. Même si les institutions internationales tendent à faire de 60 ans l'âge du début de la vieillesse, les différences internationales dans les niveaux de mortalité générale et surtout au niveau de l'espérance de vie à la naissance remettent en question la validité de cette norme. En fonction des spécificités contextuelles on peut distinguer plusieurs catégories parmi les personnes âgées, notamment les personnes du troisième âge, du quatrième âge, les retraités, etc. Toutes ces nuances posent d'emblée des problèmes fondamentaux de définition, à savoir, qui devrait être considéré comme personne âgée en Afrique.

Pour les Nations Unies, l'âge seuil de la vieillesse est généralement de 60 ans. Dans la même perspective, les pays développés ont adopté, selon leurs expériences démographiques, cette base pour les recherches et activités gérontologiques ou gériatriques. Face au vieillissement de leur population et au rallongement de la durée de vie ou à la valorisation des compétences des retraités, ces pays distinguent aujourd'hui le troisième du quatrième âge. La situation est différente dans les pays à bas revenus et à faible médicalisation où très peu de personnes survivent jusqu'à 60 ans, surtout depuis l'émergence du SIDA. Dans ce sens, le vieillissement, bien qu'universel, est contextuellement défini. Dans les pays en développement, plusieurs paramètres contextuels entrent en jeu dans la définition des personnes âgées. Le premier paramètre, démographique, est celui de l'espérance de vie à la naissance. Au Cameroun, il est de 54,3 ans (EDS 1998 : 3), alors que la moyenne de l'espérance de vie dans les pays développés est de 76 ans (Nations-Unies, 2003). Cette durée de vie reste inférieure à la moyenne mondiale et se rétrécit considérablement aux âges plus avancés. Nombreuses sont les familles qui n'ont plus la chance de voir leurs parents vivre au-delà de 90 ans. Deuxièmement, la définition du vieillissement doit prendre en compte 'l'âge où l'on sort théoriquement de l'activité de production'" (EVINA AKAM et RANDRIAMBANONA, 1988: 2). L'âge médian de la retraite ou de la cessation d'activité au Cameroun est de 55 ans. Il est de 50 ans pour les agents de l'Etat et 60 pour certains cadres des administrations publiques et privées. Cette limite est fonctionnellement dépendante du premier paramètre sus-évoqué mais aussi de la faible espérance de vie à plus de cinquante ans. Dans un contexte où les hommes sont les plus nombreux dans l'espace public, la définition doit également prendre en compte la limite d'âge de fécondabilité des femmes. Socialement, les personnes âgées sont perçues comme toutes celles dont l'âge est supérieur à la cinquantaine. Compte tenu de tous ces paramètres, nous considérons comme personnes âgées toutes celles dont l'âge est supérieur ou égal à 50 ans. 
MVEING Séraphin, FOMEKONG Félicien: Conditions de vie des ménages et recours aux soins parmi les personnes âgées au Cameroun

\section{Données et méthodes d'analyse}

Les données utilisées ont été collectées par l'Institut National de la Statistique du Cameroun en 2001 dans le cadre de la deuxième enquête camerounaise auprès des ménages (ECAM2). L'objectif principal était de produire les indicateurs sur la pauvreté et les conditions de vie des populations. L'un des modules de cette enquête permettait de recueillir les informations sur la dernière consultation de santé et cherchait à évaluer l'accès aux soins de santé. Ces données sont exploitées à partir des méthodes d'analyse descriptive bivariée et explicative multivariée en recourant à la régression logistique simple. Ainsi, il est présenté dans un premier temps quelques caractéristiques des personnes âgées (sexe, situation matrimoniale, religion, niveau d'instruction, situation d'activité, etc.) et des ménages dans lesquels ils vivent (taille et niveau de vie). Ensuite, nous évaluons le taux de morbidité en relation avec les caractéristiques individuelles et familiales. Enfin, nous examinons les interrelations entre caractéristiques des personnes âgées et de leur ménage et le recours aux soins de santé.

\section{Résultats}

Caractéristiques des personnes âgées

Le tableau 1 ci-après décrit les caractéristiques sociodémographiques et socioéconomiques des personnes âgées de 50 ans et plus, et caractérise leur cadre de vie.

Tableau 1 : Répartition des personnes de 50 ans et plus selon leurs caractéristiques socio- démographiques,

\begin{tabular}{|l|c|c|}
\hline Caractéristiques & Effectif & Pourcentage \\
\hline Milieu de résidence & 480873 & 29,1 \\
Urbain & 1170633 & 70,9 \\
Rural & 784652 & \\
\hline Sexe & 866854 & 47,5 \\
Masculin & 70207 & 52,5 \\
Féminin & 663072 & 4,3 \\
\hline Situation matrimoniale & 377860 & 40,1 \\
Célibataire & 520665 & 22,9 \\
Marié monogame & 19702 & 31,5 \\
Marié polygame & & 1,2 \\
Veuf/séparé... & & \\
Union libre & & \\
\hline
\end{tabular}




\begin{tabular}{|l|c|c|}
\hline Religion & 627264 & 380 \\
Catholique & 429209 & 26,0 \\
Protestant & 342915 & 20,8 \\
Musulmans & 251120 & 15,2 \\
Autres & 1227088 & \\
\hline Situation d'emploi & 409507 & 75,0 \\
A un emploi & 1065435 & 25,0 \\
N'a pas d'emploi & 411976 & 64,8 \\
\hline Niveau d'instruction & 167566 & 25,0 \\
Sans niveau & 638304 & 10,2 \\
Primaire & 1013202 & 38,6 \\
Secondaire et plus & 335806 & 61,4 \\
\hline Niveau de vie & 372024 & 20,3 \\
Pauvre & 943677 & 22,5 \\
Non pauvre & $\mathbf{1 6 5 1 5 0 6}$ & 57,1 \\
\hline Taille du ménage & & $\mathbf{1 0 0}$ \\
1-2 personnes & & \\
3-4 personnes & & \\
5 personnes et plus & & \\
\hline Ensemble & & \\
\hline
\end{tabular}

Les ménages comportant au moins un membre âgé de 50 ans ou plus sont majoritairement ruraux $(70,9 \%)$, et incluent plus de femmes $(52,5 \%)$ que d'hommes $(47,5 \%)$. Cette différence urbain/rural pourrait s'expliquer en partie par le retour au village des retraités, dans un contexte où les personnes âgées s'intègreraient plus facilement en milieu rural, espace où les solidarités mécaniques sont encore courantes. Lorsqu'on s'intéresse à la situation matrimoniale des enquêtés, on constate que sur trois, deux d'entre eux sont mariés. Les monogames $(40,1 \%)$ sont les plus nombreux. Le tiers de cette souspopulation est constitué des veuves et des personnes séparées de leur conjoint (e). Les catégories ciblées par l'étude (population des ménages comportant au moins un membre âgé de 50 ans ou plus) sont majoritairement chrétiens, puisque les catholiques et les protestants à eux deux concentrent plus de $64 \%$ de la population ; ils sont suivis par les musulmans $(20,8 \%)$ et les autres religions $(15,2 \%)$. 
MVEING Séraphin, FOMEKONG Félicien: Conditions de vie des ménages et recours aux soins parmi les personnes âgées au Cameroun

Trois quarts des enquêtés dans l'ensemble des ménages comportant au moins un membre âgé de 50 ans ou plus ont un emploi. Cette situation qui peut paraître curieuse serait la conséquence de la forte composition rurale de la population ciblée par l'étude. En effet, trouver un emploi est presque certain en milieu rural où les activités agricoles occupent généralement l'ensemble des populations. A peine un enquêté sur dix a atteint le niveau secondaire. Le quart de la population est constitué des personnes ayant arrêté leurs études au niveau primaire. On constate que près de deux enquêtés sur trois sont sans niveau d'instruction. La faible exposition des ruraux aux infrastructures scolaires, conjuguée à la forte pratique des activités agricoles par les populations rurales, justifierait plus les bas niveaux d'instruction que la présence des personnes âgées dans les ménages. Le niveau de vie n'a pas été directement saisi au moment de l'enquête. L'indicateur a été construit à partir des éléments constitutifs de la consommation et des dépense des ménages. Le niveau de pauvreté d'un ménage bien défini a été retenu comme caractéristique du niveau de pauvreté de chaque membre composant ce ménage. Compte tenu de cette catégorisation, une grande majorité des enquêtés $(61,4 \%)$ a été classée parmi les non pauvres. Plus de 3 enquêtés sur 5 vivent dans les ménages de 5 personnes ou plus. Cette propension aux ménages de taille élevée pourrait s'expliquer par la prédominance des ruraux dans la population enquêtée. Le milieu rural se caractérise généralement par la taille des ménages relativement plus élevée que celle des ménages urbains.

\section{Morbidité générale chez les personnes âgées}

Le tableau 2 résume les différences de morbidité en fonction des caractéristiques individuelles et familiales des personnes âgées. Dans l'ensemble, près de la moitié de personnes âgées d'au moins 50 ans ont été malades au cours des 2 dernières semaines. Ce niveau de morbidité varie avec certaines caractéristiques individuelles des personnes âgées et des ménages dans lesquels elles vivent. Ainsi, le fait de vivre en milieu urbain, d'être femme, d'être veuf/veuve/séparé (e) ou d'obédience catholique augmente le risque de contacter une maladie comme l'attestent les données du tableau ci-dessous. En effet, les plus faible taux de morbidité sont observés en milieu rural, chez les hommes, chez les non veufs (veuves)/séparés (es), surtout chez les célibataires et chez les non catholiques surtout chez les musulmans. Par ailleurs, avoir un emploi diminue le risque de contracter une maladie. Ainsi, selon les résultats du tableau ci-dessous, on peut noter que la proportion des personnes âgées ayant été malade au cours des 2 semaines est moins élevée chez les personnes ayant un emploi que chez ceux qui sont sans emploi. Considérant le niveau d'instruction, c'est chez les personnes ayant le niveau du primaire qu'on observe la proportion des personnes ayant été malades la plus élevée. 
Tableau 2 : Morbidité selon les caractéristiques du ménage

\begin{tabular}{|c|c|c|}
\hline Caractéristiques & Effectif & Pourcentage (\%) \\
\hline \multicolumn{3}{|l|}{ Milieu de résidence } \\
\hline Urbain & 244662 & 51,0 \\
\hline Rural & 572734 & 48,9 \\
\hline \multicolumn{3}{|l|}{ Sexe } \\
\hline Masculin & 369561 & 47,1 \\
\hline Féminin & 447835 & 51,7 \\
\hline \multicolumn{3}{|l|}{ Situation matrimoniale } \\
\hline Célibataire & 31944 & 45,9 \\
\hline Marié monogame & 309756 & 46,7 \\
\hline Marié polygame & 186125 & 49,3 \\
\hline Veuf/veuve/séparé (e) & 279823 & 53,7 \\
\hline Union libre & 817397 & 49,5 \\
\hline \multicolumn{3}{|l|}{ Religion } \\
\hline Catholique & 342438 & 54,6 \\
\hline Protestant & 217287 & 50,7 \\
\hline Musulmans & 144757 & 42,2 \\
\hline Autres & 112271 & 44,7 \\
\hline \multicolumn{3}{|l|}{ Situation d'emploi } \\
\hline A un emploi & 569458 & 46,4 \\
\hline N'a pas d'emploi & 242943 & 59,4 \\
\hline \multicolumn{3}{|l|}{ Niveau d'instruction } \\
\hline Sans niveau & 522196 & 49,0 \\
\hline Primaire & 221492 & 53,8 \\
\hline Secondaire et plus & 71013 & 49,5 \\
\hline \multicolumn{3}{|l|}{ Niveau de vie } \\
\hline Pauvre & 308152 & 48,3 \\
\hline Non pauvre & 509245 & 50,3 \\
\hline \multicolumn{3}{|l|}{ Taille du ménage } \\
\hline 1-2 personnes & 170431 & 50,8 \\
\hline 3-4 personnes & 186781 & 50,2 \\
\hline 5 personnes et plus & 460184 & 49,5 \\
\hline Ensemble & 816753 & 49,5 \\
\hline
\end{tabular}


MVEING Séraphin, FOMEKONG Félicien: Conditions de vie des ménages et recours aux soins parmi les personnes âgées au Cameroun

Contrairement aux attentes, les personnes âgées sont moins malades dans les ménages pauvres que dans les ménages non pauvres comme l'atteste les données du tableau ci-dessus, même si la différence n'est pas statistiquement significative. Par rapport à la taille du ménage, le taux de morbidité tend à se stabiliser.

\section{Choix du secteur de recours en cas de maladies}

Cette section permet de mesurer la demande des soins exprimés par la population âgée en rapport avec le recours à un secteur de soins donné. En Afrique en général, il est reconnu que lorsqu'une personne âgée est malade, elle a le choix entre plusieurs secteurs de consultation : secteur public ou parapublic, secteur privé laïc (vendeur informel de médicaments, ONG santé, clinique, tradipraticien, etc.), secteur privé confessionnel. S'il est vrai que pour le même épisode de maladie, il peut y avoir recours à plusieurs secteurs différents, seul le type le plus prépondérant déclaré par l'enquêté a été retenu lors de l'enquête. Ainsi, toute la trajectoire thérapeutique du maladen'a pas été saisie. Il ressort des résultats qu'en cas de maladie, le secteur public reste le plus sollicité par les personnes âgées $(42,2 \%)$, suivi du secteur privé laïc $(36,2 \%)$, le secteur privé confessionnel ne recevant que $19,6 \%$ de personnes. Le coût de consultation dans ces différentes structures pourrait expliquer cette tendance.

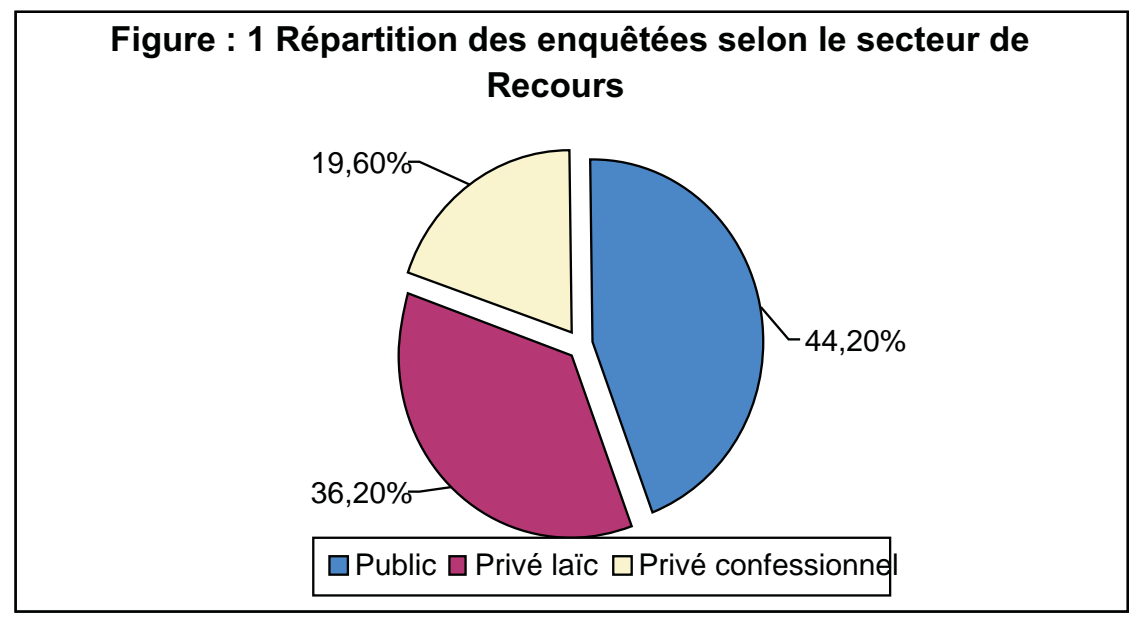

Parmi les personnes ayant choisi une structure publique, plus de $50 \%$ ont été consultées par un personnel de santé (infirmier par exemple) et $42,2 \%$ par un médecin. Il en est de même de ceux qui ont fait recours à une structure privée confessionnelle soit près de $49,6 \%$ qui on été consultés par un médecin et $44,4 \%$ par un personnel de santé. Par rapport au recours à une structure de santé privée laïque, ce sont près de $63,5 \%$ de personnes qui ont eu recours soit à un tradipraticien $(28,4 \%)$ ou à un vendeur informel de médicaments $(35,1 \%)$ indiquant de ce fait un recours fréquent à l'automédication. Cette tendance pourrait s'expliquer par le 
fait que les personnes âgées, par expérience « connaissent » leurs maladies et les médicaments pouvant les soulager. Le recours aux tradipraticiens semble peu répandu au vu de l'évolution de ce secteur au Cameroun. Néanmoins, avec la crise économique qui fragilise l'économie camerounaise depuis la fin des années 80 et qui a été suivi de la double baisse de pouvoir d'achat des ménages, ce secteur est en pleine expansion. Ces résultats ne concordent pas avec ceux de NGUINI
(1999) au Kenya. Cet auteur montre que les personnes âgées recourent moins aux services de santé modernes et plus aux consultations traditionnelles. La raison invoquée est que les services de santé modernes seraient moins efficaces pour eux (D. Duchesne, 1998). Au Bénin, au Mali et en Côte d'Ivoire, les personnes âgées semblent préférer la médecine moderne, suivi de l'automédication (AKOTO et al, 2002).

Tableau 3 : Répartition des enquêtées par secteur de soins, personnel consult ant, et motif du choix du secteur

\begin{tabular}{|l|c|c|c|c|}
\hline $\begin{array}{l}\text { Personnel consultant et motif du } \\
\text { choix de secteur }\end{array}$ & Public & Privé lä̈c & $\begin{array}{c}\text { Privé } \\
\text { confessionnel }\end{array}$ & Ensemble \\
\hline Médecin & 42,2 & 16,6 & 49,6 & 35,8 \\
Personnel de santé & 56,6 & 12,4 & 44,4 & 40,5 \\
Tradipraticien & - & 28,4 & - & 9,3 \\
Vendeur informel de médicaments & - & 35,1 & - & 11,5 \\
Autres & 1,2 & 7,5 & 6,0 & 2,9 \\
\hline Coût acceptable & 20,3 & 43,3 & 16,4 & 26,6 \\
Proximité & 39,5 & 15,0 & 25,7 & 29,2 \\
Décision familiale & 4,5 & 5,6 & 5,8 & 5,1 \\
Coutume/croyance & 0,8 & 9,8 & 2,8 & 4,0 \\
Qualité de service & 26,4 & 12,6 & 41,1 & 25,1 \\
Relations & 6,8 & 9,8 & 6,6 & 7,7 \\
Autres & 1,7 & 3,8 & 1,5 & 2,3 \\
\hline & & & & \\
\hline Total & $\mathbf{1 0 0}$ & $\mathbf{1 0 0}$ & $\mathbf{1 0 0}$ & $\mathbf{1 0 0}$ \\
\hline
\end{tabular}

En ce qui concerne les motifs avancés dans le choix du secteur de recours, on constate dans l'ensemble que, le coût de la consultation, la proximité de la structure et la qualité des services sont les principales motivations. Plus spécifiquement, le coût de la consultation a 94 été plus cité par ceux ayant fait recours à une structure privée laïque $(43,3 \%$ contre $20,3 \%$ pour ceux ayant été consulté dans une structure publique et $16,4 \%$ pour ceux ayant été consulté dans une structure privée confessionnelle). S'agissant de la proximité de la structure, c'est beaucoup 
MVEING Séraphin, FOMEKONG Félicien: Conditions de vie des ménages et recours aux soins parmi les personnes âgées au Cameroun

plus chez ceux du secteur public que ce motif a été le plus évoqué $399,5 \%$ contre $15 \%$ et $25,7 \%$ respectivement pour le secteur privé laïc et le secteur privé confessionnel). Enfin, concernant la qualité de service, elle est plus élevée chez les patients des structures privées confessionnelles $(41,1 \%$ contre $26,4 \%$ pour le secteur public et $12,6 \%$ pour le secteur privélaïc).

Caractéristiques des personnes âgées et choix du secteur de recours

Les résultats du tableau 4 cidessous montrent que les personnes âgées vivant en milieu urbain semblent préférer le secteur public que celles vivant en milieu rural, qui elles vont plus dans les structures privées. La prise en compte du sexe révèle que les femmes vont beaucoup plus vers les structures publiques que les hommes alors que les hommes semblent préférer plus les structures privées. Les célibataires vont beaucoup plus vers les structures privées confessionnelles et nettement moins dans les structures publiques et privées laïques. On note également des écarts importants entre les personnes vivant en union libre ou mariées polygames et les autres pour ce qui $\mathrm{du}$ recours aux structures publiques. C'est également le cas entre ces derniers et les autres pour ces qui est du recours aux structures privées laïques. Les musulmans recourent nettement moins aux structures privées confessionnelles que les catholiques et les protestants, pourtant, ils semblent être les plus nombreux à préférer les structures publiques et privées laïque

L'analyse en fonction des caractéristiques socio-économiques des personnes enquêtées montre que les structures publiques et les structures privées confessionnelles semblent être plus sollicitées par les personnes n'ayant aucun emploi que ceux ayant un emploi. Pour ce qui est de l'utilisation des structures privées laïques, elles sont plus fréquentées par les personnes ayant un emploi que les autres. Selon le niveau d'instruction, les personnes ayant le niveau du primaire sollicitent moins les structures publiques que ceux qui sont sans aucun niveau d'instruction et ceux ayant au moins fait le secondaire. 
Tableau 4 : Répartition des enquêtés selon les caractéristiques sociodémographiques et le secteur de recours (en \%)

\begin{tabular}{|c|c|c|c|}
\hline & Public & Privé laïc & Privé confessionnel \\
\hline \multicolumn{4}{|l|}{ Milieu de résidence } \\
\hline Urbain & 47,6 & 33,6 & 18,8 \\
\hline Rural & 42,8 & 37,3 & 19,9 \\
\hline \multicolumn{4}{|l|}{ Sexe } \\
\hline Masculin & 42,3 & 36,7 & 21,0 \\
\hline Féminin & 45,8 & 35,7 & 18,5 \\
\hline \multicolumn{4}{|c|}{ Situation matrimoniale } \\
\hline Célibataire & 41,7 & 30,8 & 27,5 \\
\hline Marié monogame & 41,7 & 36,4 & 22,0 \\
\hline Marié polygame & 48,6 & 35,5 & 16,0 \\
\hline Veuf/séparé... & 44,3 & 36,9 & 18,7 \\
\hline Union libre & 48,3 & 38,9 & 12,7 \\
\hline \multicolumn{4}{|l|}{ Religion } \\
\hline Catholique & 40,4 & 39,2 & 20,4 \\
\hline Protestant & 45,0 & 31,9 & 23,0 \\
\hline Musulmans & 46,6 & 39,5 & 13,9 \\
\hline Autres & 51,4 & 31,1 & 17,5 \\
\hline \multicolumn{4}{|l|}{ Situation d'emploi } \\
\hline A un emploi & 43,6 & 37,0 & 19,4 \\
\hline N'a pas d'emploi & 45,2 & 34,6 & 20,2 \\
\hline \multicolumn{4}{|l|}{ Niveau d'instruction } \\
\hline Sans niveau & 45,6 & 35,7 & 18,7 \\
\hline Primaire & 40,3 & 38,3 & 21,4 \\
\hline Secondaire et plus & 45,4 & 33,6 & 21,9 \\
\hline \multicolumn{4}{|l|}{ Niveau de vie } \\
\hline Pauvre & 42,1 & 39,3 & 18,6 \\
\hline Non pauvre & 45,5 & 34,3 & 20,2 \\
\hline \multicolumn{4}{|l|}{ Taille du ménage } \\
\hline 1-2 personnes & 44,0 & 33,9 & 22,1 \\
\hline 3-4 personnes & 45,8 & 32,8 & 21,4 \\
\hline 5 personnes et plus & 52,2 & 29,0 & 18,8 \\
\hline Ensemble & 44,2 & 36,2 & 19,6 \\
\hline
\end{tabular}


MVEING Séraphin, FOMEKONG Félicien: Conditions de vie des ménages et recours aux soins parmi les personnes âgées au Cameroun

Par rapport aux structures privées, les proportions les plus faibles sont observées chez les personnes ayant fait au moins le secondaire pour ce qui est des structures privées laïques et chez ceux n'ayant aucun niveau d'instruction pour ce qui est des structures privées confessionnelles. Le profil des ménages est appréhendé à travers son niveau de vie et sa taille. L'analyse a permis de mettre en exergue l'influence du niveau de vie et de la taille des ménages sur le secteur de recours. Il ressort de ces analyses que les personnes non pauvres recourent plus aux structures publiques et aux structures privées confessionnelles que les personnes pauvres qui préfèrent quant à eux les structures privées laïques. Par rapport à la taille du ménage, le recours aux structures publiques semble croître avec la taille du ménage. Par contre, pour ce qui est du recours aux structures privées (laïques et confessionnelles), c'est la tendance inverse qui est observée.

\section{Déterminants du choix du secteur de recours}

Les analyses multivariées au moyen des modèles de régressions logistiques sont utilisées pour dégager les associations statistiquement significatives entre diverses variables explicatives et chacune des trois variables dépendantes (recours au secteur public, recours au secteur privé laïc et recours au secteur privé confessionnel). Les résultats de la régression logistique montrent que selon le milieu de résidence les personnes âgées vivant en milieu rural ont plus de chances de recourir vers le secteur privé que ceux vivant en milieu urbain. Concernant le secteur public, elles ont $20 \%$ moins de chances d'y recourir que ceux du milieu urbain.

Tableau 5 : Rapports de chances associés au risque du choix du secteur de soins

\begin{tabular}{|c|c|c|c|}
\hline & \multicolumn{3}{|c|}{ Odds ratios } \\
\hline & Public & Privé laïc & Privé confessionnel \\
\hline \multicolumn{4}{|l|}{ Milieu de résidence } \\
\hline Urbain (ref) & 1 & 1 & 1 \\
\hline Rural & $0,8 *$ & $1,1 *$ & $1,2 *$ \\
\hline \multicolumn{4}{|l|}{ Sexe } \\
\hline Masculin (ref) & 1 & 1 & 1 \\
\hline Féminin & $1,2 *$ & $0,9 *$ & $0,8 *$ \\
\hline \multicolumn{4}{|l|}{ Situation matrimoniale } \\
\hline Célibataire (ref) & 1 & 1 & 1 \\
\hline Marié monogame & $1,1 \mathrm{~ns}$ & $1,3 *$ & $0,7 *$ \\
\hline Marié polygame & $1,3 *$ & $1,2 *$ & $0,5^{*}$ \\
\hline Veuf (ve)/divorcé (e)/séparé (e) & $1,1 \mathrm{~ns}$ & $1,4^{*}$ & $0,6^{*}$ \\
\hline Union libre & $1,4 *$ & $1,4^{*}$ & $0,3 *$ \\
\hline
\end{tabular}




\begin{tabular}{|c|c|c|c|}
\hline Religion & & & \\
\hline Catholique (ref) & 1 & 1 & 1 \\
\hline Protestant (e) & $1,1^{*}$ & $0,8 *$ & $1,3^{*}$ \\
\hline Musulman (e) & $1,1 *$ & $1,2 *$ & $0,7 *$ \\
\hline Niveau d'instruction & & & \\
\hline Sans niveau (ref) & 1 & 1 & 1 \\
\hline Primaire & $0,8^{*}$ & $1,2 *$ & $1,1 \mathrm{~ns}$ \\
\hline Secondaire et plus & $0,9 *$ & $1,1^{*}$ & $0,9 *$ \\
\hline Situation d'emploi & & & \\
\hline A un emploi (ref) & 1 & 1 & 1 \\
\hline N'a pas d'emploi & $1,1^{*}$ & $0,9 *$ & $1,1^{*}$ \\
\hline Niveau de vie & & & \\
\hline Non pauvre (ref) & 1 & 1 & 1 \\
\hline Pauvre & $0,8^{*}$ & $1,4 *$ & $0,9^{*}$ \\
\hline Taille du ménage & & & \\
\hline 1-2 personnes & $0,9 *$ & 1,2 & $0,9 *$ \\
\hline 3-4 personnes & $0,8^{*}$ & 1,3 & $0,9 \mathrm{~ns}$ \\
\hline 5 personnes et plus (ref) & 1 & 1 & 1 \\
\hline
\end{tabular}

$N B:$ ns non $=$ significatif $; * 1 \%$

Selon le sexe, on note que les femmes âgées ont moins de chances de recourir au secteur privé (laïc ou confessionnel) que les hommes. Elles ont par contre 1,2 fois plus de chances de recourir au secteur public que ces derniers. Les résultats montrent aussi que les mariées polygames et ceux qui vivent en union libre ont respectivement 1,3 et 1,4 fois plus de chances de recourir au secteur public que les célibataires. S'agissant du recours au secteur privé laïc, ont note que toutes les autres personnes ont nettement plus de chances d'y faire recours que les célibataires. Par rapport au secteur privé confessionnel, les célibataires y font plus recours que les autres. Les protestants et les musulmans ont légèrement plus de chances de recourir au secteur public que les catholiques. Pour ce qui est du recours au secteur privé, on constate que les protestants ont moins de chances de recourir au secteur privé laïc et plus de chance pour ce qui est du secteur privé confessionnel que les catholiques. Concernant les musulmans, c'est la tendance inverse qui est observée. Les personnes ayant au moins fait le primaire ont moins de chances de recourir au secteur public que ceux qui n'on aucun niveau 
MVEING Séraphin, FOMEKONG Félicien: Conditions de vie des ménages et recours aux soins parmi les personnes âgées au Cameroun

d'instruction. Pour ce qui est du recours au secteur privé, surtout laïc, ces derniers ont plus de chances que les sans niveau. Les personnes sans emploi ont légèrement plus de chances de recourir au secteur public et au secteur privé confessionnel que ceux qui ont un emploi. Pour ce qui est du recours au secteur privé laïc, c'est la tendance inverse qui est observée. Les pauvres ont moins de chances de recourir au secteur public et au secteur privé confessionnel que les non pauvres. On note cependant qu'ils ont 1,4 fois plus de chances de recourir au secteur privé laïc que les non pauvres. Selon la taille du ménage, on constate que plus le ménage est petit, plus les personnes âgées qui y vivent ont moins de chances de recourir au secteur public et plus, elles ont la chance de recourir au secteur privé laïc.

Les résultats ci-dessus montrent que même si les conditions de vie des personnes âgées à travers le profil des ménages dans lesquels elles vivent ainsi que de leurs caractéristiques individuelles influencent significativement leur comportement thérapeutique en termes du choix du secteur de recours aux soins, leur poids dans l'explication de ce choix reste faible. On peut donc penser à une influence d'autres facteurs tels que les facteurs institutionnels et les croyances étiologiques.

Facteurs institutionnels et croyances étiologiques

Le vieillissement dans les pays en développement survient général dans un contexte de précarité économique et institutionnelle qui limite les possibilités d'assistance et d'offre de services spécialisés aux questions de gériatrie ou de gérontologie. Comme dit plus haut, la préoccupation des pouvoirs publics est plus souvent tournée vers les problèmes liés à la jeunesse, à la femme, à la pauvreté, etc. Au Cameroun, le vieillissement ne constitue pas à proprement parler un problème, ce qui limite les réponses que la société et l'Etat apportent aux besoins sanitaires spécifiques des personnes âgées dépendantes, surtout en terme d'accès à divers secteurs de soins.

Les croyances étiologiques sont aussi importantes. Dans l'imaginaire collectif, les personnes âgées ne sont pas trop exposées aux maladies. Elles sont plutôt perçues comme victimes de leur âge avancé, qui entraîne un affaiblissement du système immunitaire. Dans cette logique, la vieillesse est en soi une maladie. Dès lors, en cas de maladie, ils sont souvent très peu conduits dans les services hospitaliers. On considère parfois les personnes âgées comme des "morts vivants", c'est-à-dire des personnes pour qui le recours aux soins de santé ont peu d'importance dans la mesure "ils n'attendent plus que la mort...quel que soit ce qu'on leur donnera comme soins, çà ne rallongera plus leurs jours. Ils savent eux-mêmes et parfois ils vous disent de ne plus vous casser pour eux, mais d'investir sur les jeunes enfants car la famille peut plus compter sur ces derniers que sur les vieillards" (extrait d'un entretien réalisé en février 2005 par FOMEKONG et MIMCHE auprès d'une case de retraite à SIMBOCKYaoundé). Le fait de concevoir les dépenses allouées aux soins des personnes âgées comme des investissements improductifs 
limite la demande et l'accès aux soins dans les services spécialisés et justifie davantage le recours à l'automédication familiale. Ce background culturel joue en défaveur des recours aux secteurs modernes des soins par les familles des personnes âgées dans la mesure où elles deviennent des personnes dépendantes. Ces analyses montrent l'influence de la construction sociale et culturelle de la maladie sur l'accessibilité culturelle aux soins de santé au sein de toute la communauté dans la mesure où chaque population a sa manière de vivre et de penser sa santé, de se soigner qui lui semble idéale en fonction de certains repères socioculturels. Ce sont ces croyances étiologiques qui influencent également la demande de soins des personnes âgées et oriente le choix des secteurs de recours au sein des familles camerounaises.

\section{Discussion et conclusion}

$\mathrm{Au}$ terme de cette analyse des comportements thérapeutiques des personnes âgées, il ressort un pluralisme thérapeutique qui se reflète dans la diversité de recours aux différents secteurs de soins et les logiques sous-jacentes. Le recours aux soins est multiple et parfois même simultané, oscillant entre tradition et modernité. Les recours et itinéraires thérapeutiques vont de pair avec les croyances étiologiques, notamment la perception de la vieillesse elle-même comme une maladie, et ceci autant par les personnes âgées elles mêmes que par leur réseau social. Les recours thérapeutiques varient en fonction des caractéristiques sociodémographiques des vieillards, leur niveau de dépendance et des conditions de vie de leur ménage. Notre étude suggère enfin que l'importance accordée aux soins modernes n'indique pas un rejet de la tradition. Au total, un plaidoyer en faveur d'un plan de prise en charge gratuite des personnes âgées à l'instar du plan SESAME (depuis le 1er septembre 2006) au Sénégal devrait aussi être engagé au Cameroun avec un accent particulier dans le milieu rural. Parallèlement, le Cameroun devrait continuer sa lutte contre la pauvreté des ménages par des politiques robustes de retraite active et renforcer le cadre institutionnel de lutte contre la discrimination et l'exclusion qui a plus de conséquences sur les couches vulnérables et notamment les personnes âgées.

\section{Références Bibliographiques}

AERTS, J.-J. et al., L'économie camerounaise. Un espace évanoui, Paris, Karthala, 2000, 287p.

AKOTO, E. M. et al., Se soigner aujourd'hui en Afrique de l'Ouest: pluralisme thérapeutique entre traditions et modernité (Bénin, Côte d'Ivoire et Mali), Les Cahiers de l'IFORD, ${ }^{\circ} 27$, 2002,169p.

ANDREWS, G. " Research direction in the region : past, present and future", in Philips, R.David, Ageing in East and South-East Asia, London, Edward Arnold, 1992. 
MVEING Séraphin, FOMEKONG Félicien: Conditions de vie des ménages et recours aux soins parmi les personnes âgées au Cameroun

BARBIER, J. C., COURADE G. et GUBRY, P., "L'exode rural au Cameroun », Cahier ORSTOM, Série Sciences Humaines, Vol. 18, n¹,1981-82, pp. 107-147

BARBIER, J. C. COURADE, G. et GUBRY, P. , L'exode rural au Cameroun, Collection Travaux et documents del'ISH, n¹1,1978,112 p.

COURADE, G. (sous la direction), Le village camerounais à l'heure de l'ajustement, Paris, Karthala, 1994.

DESSOUANE, P. et VERRE, P. "Cameroun: du développement autocentré au nationallibéralisme", pp.111-119, in Le réveil du Cameroun, Politique africaine $\mathrm{n}^{\circ} 22$, Paris, Karthala, 1986.

Duchèsne, D ; Evaluation de la fonction de demande de soins de santé en Tanzanie, CRDE, Uninersité de montréal, Octobre 1998 Enquête Démographique et de Santé 1998, BUCREP/MINPAT

EGGERICKX, Th. et TABUTIN, D. (Nov. 2001) Le vieillissement démographique dans le monde. Historique, mécanismes et tendances, Université catholique de Louvain, Département des Sciences de la Population et du développement, Document de travail $n^{\circ} 14$.

EVINA AKAM et RANDRIAMBANONA, R., Vieillissement et besoins éducationnels des personnes âgées. Le cas spécifique de l'Afrique,
Communication présentée à la rencontre d'experts organisée par le BIT, l'AIUTA, l'IEIAS et l'AIDAC, Genève, 8-10 décembre 1987, Document de recherche $n^{\circ} 86$, Institut de démographie/ Université Catholique de Louvain, Janvier 1988

EVINA AKAM, Interview réalisée dans le dossier de Récup'Action $\mathrm{n}^{\circ} 006$, décembre 2003

FOMEKONG Félicien ; Influence des caractéristiques démographiques et socio-économiques sur la prise en charge du paludisme dans uns plantation du Sud Cameroun (Hévécam), Mémoire DESSD, Janvier 2004

FOMEKONG Félicien, Honoré MIMCHE, Le vieillissement de la population en Afrique subsaharienne. Quels défis pour les politiques sociales et de population ? in revue Internationale des Sciences Humaines et Sociales, Vol1, No 1 Août 2006, Yaoundé-Cameroun

GENDREAU, F., Démographie africaine. Perspectives sur l'an 2000, AUPELF/UREF.

HENRARD, J.-C., Le système de soins et d'aide aux personnes âgées , pp. 2135 , in Plan urbain

et MIRE, Vieillir dans la ville, Paris, L'Harmattan, Collection «Villes et Entreprises » Ministère de la santé 
publique, 1990, Carte sanitaire $d u$ Cameroun.

Nations-Unies, Perspectives de la population dans le monde 2002, Population Reference Bureau, 2003

NGUINI, R : Health seeking behaviour in the reform process for rural household: the case of Mwea division, Kiringaga district, AERC Research paper 95, Nairobi, Mars 1999
OMS, Gouvernement du Mali, UNICEF, 1999, Rapport sur la revue de la mise en oeuvre de l'Initiative de Bamako, Bamako, 8-12 mars 1999.

OMS : Vieillir en restant actif : cadre d'orientation, 2002. 